\title{
Activité antioxydante de quelques plantes utilisées dans la région de Tiassalé (Côte d'Ivoire) dans le maintien de la santé de la peau
}

\author{
Koffi Akessé Georges, Doctorant \\ Ahoua Angora Remi Constant, Docteur \\ UFR Sciences de la Nature, \\ Université Nangui Abrogoua, Abidjan,Côte d'Ivoire \\ Ekou Lynda, Maître de Conférences \\ Ekou Tchirioua, Professeur Titulaire \\ UFR Sciences Fondamentales et Appliquées, \\ Université Nangui Abrogoua, Abidjan, Côte d'Ivoire
}

Kone Mamidou Witabouna, Professeur Titulaire

UFR Sciences de la Nature, Université Nangui Abrogoua, Abidjan, Côte d'Ivoire/ Centre Suisse de Recherches Scientifiques en Côte d'Ivoire, Abidjan, Côte d'Ivoire

Doi: 10.19044/esj.2018.v14n30p338 URL:http://dx.doi.org/10.19044/esj.2018.v14n30p338

\begin{abstract}
Skin health is a concern for many people. This aim of this study is to evaluate in vitro the antioxidant activity of 10 plants used in cosmetopoeia for the maintenance of skin health. To achieve this objective, 30 extracts were tested using 2,2'-diphenyl-1-picrylhydrazyl radical (DPPH) and 2,2'-azino-bis (3-ethylbenzothiazoline-6) acid. -sulfonic) (ABTS + •) assays. The extracts studied were rich in total polyphenols, flavonoids, gallic tannins and alkaloids. For the DPPH reduction, 7 extracts including 5 methanolic and 2 aqueous extracts showed a percentage of inhibition similar to that of Vitamin C (95.79 $\pm 10.53 \%$ ) used as a control. With ABTS, 11 extracts ( 5 methanol, 3 aqueous and 3 hexane) gave reduction percentages close to Trolox $(95.97 \pm 8.51 \%)$. These active plants could play a role in the maintaining of skin.
\end{abstract}

Keywords: Oxidative stress, antioxidant activity, skin, phytocompounds, medicinal plants, Côte d'Ivoire

\section{Résumé}

La santé de la peau constitue une préoccupation pour de nombreuses personnes. Ainsi, ce travail a pour objectif d'évaluer, in vitro l'activité 
antioxydante de 10 plantes utilisées en cosmétopée dans le maintien de la santé de la peau. Pour cela, 30 extraits ont été testés en utilisant les tests de réduction des radicaux 2,2'-diphényl-1-picrylhydrazyle (DPPH) et de 1'acide 2,2'-azino-bis-(3-éthylbenzothiazoline-6-sulfonique) (ABTS+•). Le criblage phytochimique montre que les extraits étudiés sont riches en polyphénols totaux, en flavonoïdes, en tanins galliques et alcaloïdes. Pour la réduction du DPPH, sept extraits dont cinq extraits méthanoliques et deux aqueux ont montré un pourcentage d'inhibition superposable à celui de la Vitamine $\mathrm{C}$ $(95,79 \pm 10,53 \%)$ utilisé comme contrôle. Avec l'ABTS, 11 extraits (5 au méthanol, 3 aqueux et 3 à l'hexane) ont donné des pourcentages de réduction sensiblement proches de celui du Trolox $(95,97 \pm 8,51 \%)$. Ces plantes actives pourraient jouer un rôle dans le maintien de la peau.

Mots-clés : Stress oxydant, activité antioxydante, peau, phytocomposés, plantes médicinales, Côte d'Ivoire

\section{Introduction}

La santé de la peau demeure toujours un problème d'actualité et de santé publique dans un contexte où la peau est soumise aux agressions extérieures. Les affections cutanées entre autres les mycoses, vergetures, eczéma, acné et le vieillissement d'origine multifactorielle sont des problèmes importants de la peau. Le stress oxydant est l'une des principales causes aggravantes de ces affections. C'est l'un des facteurs potentialisant des troubles cutanés qui surviennent surtout avec l'âge ainsi que le vieillissement des moyens de défense de l'organisme (Favier, 2013). Les maladies de la peau ont un impact social négatif sur la vie des personnes qu'elles affectent. Les estimations de la prévalence, pour ces maladies sont alarmantes. En France, la Société de Dermatologie chiffre à 15 millions, les adolescents et les femmes adultes qui souffrent d'acné (Bhate et al., 2013). Dans les pays tropicaux comme le Sénégal, les dermatoses touchent $30 \%$ de la population en milieu rural (Léon et al., 2014). En Côte d'Ivoire, les prévalences déjà rapportées pour les mycoses (teignes) se situaient entre 58 à $96 \%$ dans la tranche d'âge de 5 à 9 ans (Attoh-Touré et al., 2009). Ces chiffres sont inquiétants. De plus, l'application topique des antioxydants de synthèse comme le benzoate de benzyle utilisés depuis des décennies pour réduire les dégâts du stress oxydant s'est avérée responsable d'effets indésirables pour la peau (Brieger et al., 2012).

La recherche de nouvelles alternatives pour le maintien de la santé de la peau s'avère nécessaire. Dans cette quête se trouve la cosmétopée où les plantes à usages cosmétiques sont de plus en plus sollicitées. Elles sont utilisées depuis la nuit des temps dans les soins des cheveux, la beauté corporelle et pour se parfumer. Plusieurs d'entre elles et leurs métabolites 
secondaires sont connus pour leurs effets antioxydants naturels, antiseptiques, etc. Les polyphénols, les tanins, les alcaloïdes, les huiles essentielles sont responsables de l'activité antioxydant des plantes (Békro et al., 2013). Les antioxydants naturels suscitent donc de plus en plus un intérêt.

L'objectif de cette étude est d'évaluer in vitro, l'activité antioxydante de 10 plantes utilisées en cosmétopée dans la région de Tiassalé (Sud Côte d'Ivoire) pour améliorer la santé de la peau.

\section{Matériel et méthodes}

\section{Matériel végétal}

Dix plantes médicinales issues de la région de Tiassalé (sud Côte d'Ivoire) ont été utilisées pour l'étude. Cette région regorge d'une grande diversité floristique. Par ailleurs, les espèces végétales sélectionnées étaient parmi celles les plus citées par les populations pour leur usage en cosmétopée.

\section{Préparation des extraits végétaux}

Les organes de plantes récoltés ont été séchés à $18{ }^{\circ} \mathrm{C}$ (sous climatisation) puis pulvérisés à l'aide d'une broyeuse.

L'hexane, le méthanol et l'eau ont été utilisés comme solvants d'extraction. Pour les extraits aqueux, une quantité de $20 \mathrm{~g}$ de poudre a été macérée dans $200 \mathrm{~mL}$ d'eau distillée.

Le mélange a été soumis à une agitation mécanique pendant $24 \mathrm{~h}$. Après filtration, les extraits ont été lyophilisés pour obtenir les extraits bruts secs.

Des extractions successives avec des solvants de polarité croissante ont été aussi réalisées. Ainsi, $20 \mathrm{~g}$ de chaque poudre a été macérée dans $200 \mathrm{~mL}$ d'hexane pendant $24 \mathrm{~h}$ sous agitation. Le macéré a été filtré sur du coton, puis du papier filtre. Après filtration et concentration à l'évaporateur rotatif à 40 ${ }^{\circ} \mathrm{C}$, les extraits hexaniques ont été obtenus. Le marc résiduel a été séché à température ambiante, pesé, puis repris dans un volume de $200 \mathrm{~mL}$ de méthanol. Le mélange a été macéré à nouveau pendant $24 \mathrm{~h}$, puis a subi les mêmes étapes de concentration et de lyophilisation.

Le rendement a été calculé en utilisant la formule suivante :

$$
\boldsymbol{R d t}(\%)=\frac{m}{M} x \mathbf{1 0 0}
$$

$\mathrm{Rdt}=$ Rendement, $\mathrm{m}=$ masse de l'extrait préparé $; \mathrm{M}=$ masse de la poudre utilisée

\section{Recherche des groupes de composés chimiques}

La recherche des groupes de composés chimiques chez les extraits bruts a été réalisée en tubes et sur Chromatographie sur Couche Mince (Wagner et al., 1987 ; Dohou et al., 2005). 
Caractérisation des saponosides

Dans un tube à essai, $10 \mathrm{ml}$ de macéré ont été agités énergétiquement pendant 15 secondes puis laissés au repos pendant $15 \mathrm{~min}$. Une hauteur de mousse persistante supérieure à $1 \mathrm{~cm}$ indique la présence de saponosides.

Analyse qualitative sur Chromatographique sur couche mince (CCM)

Des chromatogrammes sur couche mince ont été réalisés sur des plaques de gel de silice (Silicagel $60 \mathrm{~F}_{254}$ ) pour la recherche des polyphénols, des flavonoïdes, des tanins galliques ou catéchiques et des alcaloïdes. Le système d'élution est composé de chloroforme/méthanol/eau dans les proportions $65: 35: 5$.

- Révélation des phénols totaux

Le réactif de Folin-Ciocalteu a été pulvérisé sur le chromatogramme puis séché après chauffage pendant $10 \mathrm{~min}$. L'observation des spots bleus dans le visible indique la présence des polyphénols.

- Révélation des flavonoïdes

Une solution éthanolique à $5 \%$ de chlorure d'aluminium $\left(\mathrm{AlCl}_{3}\right)$ a été pulvérisée sur le chromatogramme. Le spot jaune dans le visible ou jaune-vert sous UV à 366 nm indique la présence de flavonoïdes (Merck., 1980).

- Révélation des tanins

Une solution de chlorure de fer $\left(\mathrm{FeCl}_{3}\right)$ à $10 \%$ a été pulvérisée sur le chromatogramme. Le spot bleu-noir dans le visible indique la présence des tanins galliques et la coloration vert ou vert foncé montre des tanins condensés (N'Guessan et al., 2009).

- Révélation des alcaloïdes

Après pulvérisation sur le chromatogramme du réactif de Dragendorff, l'observation dans le visible de spots orangés indique des alcaloïdes.

\section{Activité antiradicalaire des extraits végétaux préparés}

Test au 2,2'-diphényl-1-picrylhydrazyle (DPPH)

La mesure de l'activité antiradicalaire réalisée sur des extraits de plantes a été effectuée par le test au 2,2'-diphényl-1-picrylhydrazyle (DPPH) selon la méthode décrite par Sanchez-Moreno et al. (2002). Cette technique est basée sur la décoloration de la solution de DPPH par un extrait végétal actif. La Vitamine $\mathrm{C}$ a été utilisée comme témoin positif. Une gamme de concentrations $(1000,500,250,62,12,31,25,15,62$ et 7,81 $\mu \mathrm{g} / \mathrm{mL})$ des extraits de plantes ou de la vitamine $\mathrm{C}$ (antioxydant de référence) a été préparée. Un volume de $100 \mu \mathrm{l}$ d'extrait a été mélangé à $2,5 \mathrm{~mL}$ d'une solution de DPPH (0,04 \% dans du méthanol). Après homogénéisation, le mélange a 
été incubé à la température du laboratoire à l'obscurité. Après $30 \mathrm{~min}$ d'incubation, les absorbances ont été mesurées à $517 \mathrm{~nm}$ contre un « blanc » qui ne contient que du méthanol. L’expérience a été reprise trois fois.

Le pourcentage d'inhibition du radical DPPH a été calculé selon l'équation suivante :

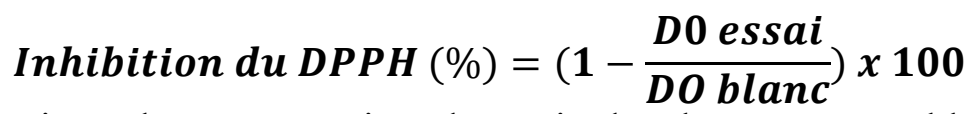

La $\mathrm{CI}_{50}$ qui est la concentration d'extrait de plante responsable de $50 \%$ d'inhibition du radical DPPH a été déterminée à partir de la courbe d'étalonnage de la vitamine $\mathrm{C}$.

Test à l'acide 2,2-azino-bis-(3-éthylbenzothiazoline-6-sulfonique) (ABTS+•)

Le potentiel des extraits à réduire le radical ABTS a été évalué à l'aide de la méthode décrite par Miller et al., (1993). Le principe est fondé sur la capacité d'un antioxydant à stabiliser le radical cationique $\mathrm{ABTS}^{\circ} \mathrm{de}$ coloration bleu-vert en le transformant en $\mathrm{ABTS}^{+}$incolore par piégeage d'un proton. Le radical cationique $\mathrm{ABTS}^{+}$a été obtenu à partir de $10 \mathrm{ml}$ d'ABTS à $2 \mathrm{mM}$ et $100 \mu \mathrm{l}$ de persulfate de potassium à $70 \mathrm{mM}$. Le mélange a été conservé à l'obscurité pendant $6 \mathrm{~h}$ avant la réalisation du test. Une gamme de concentrations $(1000,500,250,62,12,31,25,15,62$ et 7,81 $\mu \mathrm{g} / \mathrm{mL})$ des extraits végétaux ou du Trolox (antioxydant de référence) a été préparée, puis $100 \mu \mathrm{l}$ de ces solutions ont été mélangés à 2,5 ml d'une solution d'ABTS. Le mélange obtenu a été homogénéisé puis incubé à l'obscurité. Après $30 \mathrm{~min}$ d'incubation, les absorbances sont mesurées à $734 \mathrm{~nm}$. La CI50 a été déterminée graphiquement à partir de la droite d'étalonnage du Trolox. Les essais ont été répétés 3 fois.

Le pourcentage d'inhibition du radical ABTS est calculé selon l'équation suivante :

$$
\text { Inhibition de ABTS }(\%)=\left(1-\frac{\text { D0 essai }}{\text { DO blanc }}\right) x 100
$$

\section{Traitement des données et analyses statistiques}

Le logiciel STATISTICA version 7.1 a été employé pour le traitement des données des tests biologiques. Lorsqu'une différence significative est observée entre les moyennes des pourcentages d'inhibition du DPPH et de l'ABTS, l'ANOVA a été complétée par des comparaisons multiples en effectuant le test de Fischer. Ce test permet d'identifier le ou les extraits qui diffèrent significativement les uns des autres. La plus petite différence significative $(p p d s)$ entre les activités des extraits a été fixée à $p \leq 0,05$. Si $p \leq$ 0,05 on conclue que la différence est significative entre les moyennes des activités ; par contre lorsque $p \geq 0,05$, on conclue que la différence entre les moyennes des activités n'est pas significative (Dagnelie, 1999). 


\section{Résultats}

\section{Rendement et caractéristiques physico-chimiques des extraits préparés}

Les rendements obtenus variaient d'un solvant à un autre et d'une plante à une autre. Pour les extraits aqueux de toutes les plantes, les rendements sont compris entre 5,19 et 83,19\%. L'extrait aqueux de Anchomanes difformis (Blume) Engl. a donné le plus grand rendement (83,19 $\%)$. Les extraits méthanoliques ont donné des rendements, qui variaient de 0,88 à 48,70 \%. L'extrait au méthanol de Musa paradisiaca L. a présenté le rendement le plus élevé, à 48,70\%. Avec les extraits hexaniques, les rendements oscillent entre 6,06 et 46,46\%, l'extrait de Sida acuta a montré le grand rendement $(46,46 \%)$. Les extraits aqueux et méthanoliques ont donné les plus grands rendements, tandis que les plus faibles ont été obtenus avec l'hexane. La majorité des extraits préparés ont présenté un aspect très collant, ainsi qu'une couleur verte (tableau 1).

Tableau 1 : Caractéristiques physico-chimiques et rendements des espèces végétales sélectionnées (masse de poudre végétale $=20 \mathrm{~g}$ )

\begin{tabular}{|c|c|c|c|c|c|}
\hline Espèces végétales & $\begin{array}{l}\text { Organes } \\
\text { testés }\end{array}$ & $\begin{array}{l}\text { Extraits } \\
\text { végétaux }\end{array}$ & $\begin{array}{l}\begin{array}{l}\text { Masses } \\
(\mathrm{g})\end{array} \\
\end{array}$ & $\begin{array}{l}\text { Rendemen } \\
\mathrm{t}(\%)\end{array}$ & Aspect/Couleur \\
\hline $\begin{array}{l}\text { Anchomanes } \\
\text { (Blume) Enol }\end{array}$ & Feuilles & EA & 0.94 & 83.19 & collant/vert \\
\hline \multirow{2}{*}{ (Blume) Engl. } & & EM & 0,01 & 0,88 & collant/vert \\
\hline & & $\mathrm{EH}$ & 0,18 & 15,93 & collant/marron \\
\hline \multirow[t]{3}{*}{ Cajanus cajan $\mathrm{L}$. } & Feuilles & EA & 0,9 & 45,45 & flocon/jaune \\
\hline & & EM & 0,49 & 24,75 & flocon /jaune \\
\hline & & EH & 0,59 & 29,80 & flocon /jaune \\
\hline \multirow{3}{*}{ Cyathula prostata $\mathrm{L}$. } & $\begin{array}{l}\text { Plante } \\
\text { entière }\end{array}$ & EA & 1,8 & 56,24 & pâte/marron \\
\hline & Fruit & EM & 0,94 & 29,38 & pâte/marron \\
\hline & Fruit & EH & 0,46 & 14,38 & pâte/marron \\
\hline \multirow[t]{3}{*}{ Elaeis guineensis (Jacq.) } & Racines & EA & 1,4 & 70,71 & poudre/marron \\
\hline & & EM & 0,24 & 12,12 & collant/marron \\
\hline & & $\mathrm{EH}$ & 0,34 & 17,17 & collant/marron \\
\hline \multirow[t]{3}{*}{ Eleusine indica (L.) Gaertn } & Racines & EA & 0,96 & 48,49 & poudreux/blanche \\
\hline & & EM & 0,9 & 45,45 & poudreux/blanche \\
\hline & & $\mathrm{EH}$ & 0,12 & 06,06 & poudreux/blanche \\
\hline \multirow[t]{3}{*}{ Mimosa pudica L. } & $\begin{array}{l}\text { Plante } \\
\text { entière }\end{array}$ & EA & 0,54 & 22,78 & collant/jaune \\
\hline & & EM & 0,92 & 38,82 & collant/jaune \\
\hline & & $\mathrm{EH}$ & 0,91 & 38,40 & collant/jaune \\
\hline \multirow[t]{3}{*}{ Musa paradisiaca $\mathrm{L}$. } & Feuilles & EA & 0,92 & 46,46 & pâte/marron \\
\hline & & EM & 0,94 & 48,70 & pâte/marron \\
\hline & & $\mathrm{EH}$ & 0,14 & 07,08 & pâte/marron \\
\hline \multirow[t]{3}{*}{ Nephrolepis bisserata L. } & Feuilles & EA & 1,05 & 65,22 & pâte/vert \\
\hline & & EM & 0,1 & 06,21 & pâte/vert \\
\hline & & $\mathrm{EH}$ & 0,46 & 28,57 & pâte/vert \\
\hline
\end{tabular}




\begin{tabular}{|c|c|c|c|c|c|}
\hline Sida acuta Burm. f. & $\begin{array}{l}\text { Ecorce de } \\
\text { tronc }\end{array}$ & EA & 0,1 & 05,19 & collant/vert \\
\hline \multirow{5}{*}{$\begin{array}{l}\text { Triplochiton scleroxylon } \\
\text { Schum. }\end{array}$} & \multirow{5}{*}{$\begin{array}{l}\text { Ecorce de } \\
\text { tronc }\end{array}$} & EM & 0,89 & 46,11 & collant/vert \\
\hline & & $\mathrm{EH}$ & 0,92 & 46,46 & collant/vert \\
\hline & & EA) & 0,98 & 58,68 & pâte/jaune \\
\hline & & EM & 0,15 & 08,98 & pâte/jaune \\
\hline & & $\mathrm{EH}$ & 0,54 & 32,34 & pâte/jaune \\
\hline
\end{tabular}

$\mathrm{EA}=$ extrait aqueux $; \mathrm{EM}=$ extrait méthanolique $; \mathrm{EH}=$ extrait hexanique

\section{Composés chimiques détectés chez les plantes étudiées}

Le profil chromatographique sur CCM a révélé une diversité des composés chimiques, notamment des polyphénols, des flavonoïdes, des tanins et des alcaloïdes chez les cinq plantes étudiées. Chez les extraits méthanoliques de E. guineensis et $C$. cajan, les polyphénols $\left(\mathrm{R}_{\mathrm{f}}=0,53\right)$, les flavonoïdes $\left(R_{\mathrm{f}}=0,66\right)$ ont été révélés par le réactif de Folin-Ciocalteu et de $\mathrm{AlCl}_{3}$ respectivement. En plus, des tanins $\left(\mathrm{R}_{\mathrm{f}}=0,26\right)$ et des alcaloïdes $\left(\mathrm{R}_{\mathrm{f}}=\right.$ $0,46)$ ont été détectés dans l'extrait méthanolique de E. guineensis. Quant aux flavonoïdes $\left(\mathrm{R}_{\mathrm{f}}=0,26\right)$, ils ont été révélés dans l'extrait aqueux de $C$. cajan $\left(R_{\mathrm{f}}=0,50\right)$. Les concentrations inhibitrices des extraits végétaux testés et les groupes de composés chimiques sont consignés dans le tableau 2.

\section{Activités antioxydantes des extraits}

Activité antiradicalaire au DPPH

Sur un total de 30 extraits évalués pour leur pouvoir réducteur du $\mathrm{DPPH}$, sept extraits dont cinq méthanoliques et deux aqueux ont donné un pourcentage d'inhibition superposable à celui de la Vitamine C $(95,79 \pm 10,53$ $\%)$ utilisé comme contrôle. La comparaison des moyennes des pourcentages d'inhibition a montré que tous les extraits au méthanol de $C$. cajan, de $C$. prostata, de E. indica, de E. guineensis, et de A. difformis possèdent un pouvoir antioxydant. Cependant, les extraits méthanoliques de $C$. cajan (95,71 $\pm 9,67 \%)$, de $C$. prostata $(94,91 \pm 7,04 \%)$, de $E$. indica $(93,54 \pm 10,21 \%)$ ont donné les plus fortes activités. E. guineensis $(89,02 \pm 12,76 \%)$, et $A$. difformis $(87,19 \pm 10,54 \%)$ ont eu des activités intermédiaires. Les $\mathrm{CI}_{50}$ sont comprises entre 27,20 , et $60 \mu \mathrm{g} / \mathrm{mL}$. Ces extraits sont donc moins actifs que la Vitamine C $(8,34 \mu \mathrm{g} / \mathrm{mL})$. La différence entre les moyennes des pourcentages d'inhibition des extraits aqueux de E. guineensis $(89,02 \pm 12,76$ $\%)$, de $A$. difformis $(88,54 \pm 7,04 \%)$ est non significative $(p>0,05)$. Les extraits aqueux de $A$. difformis $(88,54 \pm 7,04 \%)$ et de C. prostata $(74,90 \pm 7,04 \%)$, quant à eux engendrent des activités intermédiaires. Ces extraits sont donc moins actifs que la Vitamine C $(96,79 \pm 10,53 \%)$. 
Tableau 2 : Types de composés chimiques détectés sur CCM

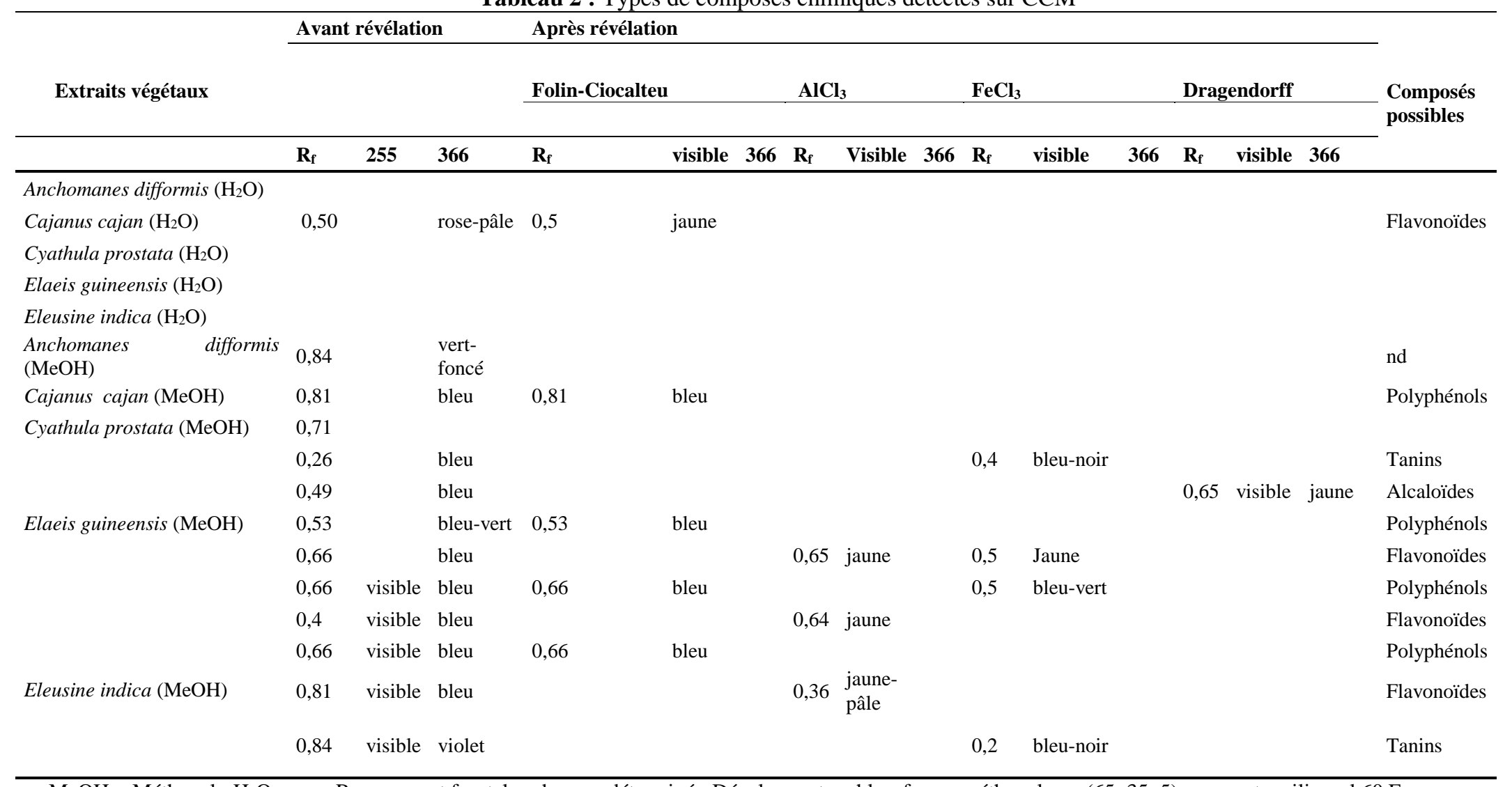

$\mathrm{MeOH}=$ Méthanol $; \mathrm{H}_{2} \mathrm{O}=$ eau, $\mathrm{R}_{\mathrm{f}}=$ rapport frontal $; \mathrm{nd}=$ non déterminé $;$ Développant = chloroforme-méthanol-eau $(65$ :35 :5) ; support = silicagel 60 F 254 
Leurs valeurs de $\mathrm{CI}_{50}$ sont comprises entre 45,80 et $30,00 \mu \mathrm{g} / \mathrm{mL}$. En revanche, aucun extrait à l'hexane testé n'a montré une activité antioxydante. Dans l'ensemble, les extraits au méthanol ont été plus actifs sur le radical libre DPPH que ceux aqueux (tableau 3).

Tableau 3 : Pourcentages d'inhibition du DPPH des espèces végétales

\begin{tabular}{llll}
\hline Espèces végétales et témoin & Extraits méthanoliques & $\begin{array}{l}\text { Extraits } \\
\text { hexaniques }\end{array}$ & Extraits aqueux \\
\hline Anchomanes difformis & $87,19 \pm 10,54^{\mathrm{a}}$ & $15,00 \pm 2,30^{\mathrm{de}}$ & $88,54 \pm 7,04^{\mathrm{a}}$ \\
Cajanus cajan & $95,71 \pm 9,67^{\mathrm{a}}$ & $17,53 \pm 3,04^{\mathrm{de}}$ & $37,86 \pm 4,38^{\mathrm{c}}$ \\
Cyathula prostata & $94,91 \pm 7,04^{\mathrm{a}}$ & $19,18 \pm 4,24^{\mathrm{de}}$ & $74,90 \pm 7,04^{\mathrm{b}}$ \\
Elaeis guineensis & $89,02 \pm 12,76^{\mathrm{a}}$ & $13,69 \pm 2,14^{\mathrm{de}}$ & $14,30 \pm 1,45^{\mathrm{de}}$ \\
Eleusine indica & $93,54 \pm 10,21^{\mathrm{a}}$ & $18,84 \pm 1,05^{\mathrm{de}}$ & $18,84 \pm 1,05^{\mathrm{de}}$ \\
Mimosa pudica & $9,34 \pm 1,01^{\mathrm{de}}$ & $10,74 \pm 1,21^{\mathrm{de}}$ & $10,42 \pm 1,04^{\mathrm{de}}$ \\
Musa paradisiaca & $8,18 \pm 1,28^{\mathrm{de}}$ & $8,23 \pm 1,27^{\mathrm{de}}$ & $7,61 \pm 1,17^{\mathrm{de}}$ \\
Nephrolepis bisserata & $6,04 \pm 1,41^{\mathrm{de}}$ & $9,69 \pm 1,04^{\mathrm{de}}$ & $8,50 \pm 1,42^{\mathrm{de}}$ \\
Sida acuta & $9,91 \pm 1,22^{\mathrm{de}}$ & $10,25 \pm 0,55^{\mathrm{de}}$ & $9,71 \pm 0,70^{\mathrm{de}}$ \\
Triplochiton scleroxylon & $8,64 \pm 1,10^{\mathrm{de}}$ & $10,25 \pm 0,55^{\mathrm{de}}$ & $9,46 \pm 1,73^{\mathrm{de}}$ \\
\hline
\end{tabular}

Vitamine C (témoin) $95,79 \pm 10,53^{\mathrm{a}}$

Paramètres statistiques $\mathrm{F}(30,279)=17,614: p=0,000$

Les valeurs avec les mêmes lettres en exposant dans la même colonne ne sont pas statistiquement différentes.

${ }^{\mathrm{a}}$ : activité forte, ${ }^{\mathrm{b}}$ : Activité intermédiaire, de c: Activité faible

Activité antiradicalaire à l'ABTS

Avec l'ABTS, 11 extraits ont montré une activité antiradicalaire (tableau 4). Toutefois, deux extraits au méthanol, un aqueux et trois à l'hexane ont donné des pourcentages de réduction proches à celui du Trolox $(95,97 \pm 8,51 \%)$. La comparaison des moyennes des pourcentages d'inhibition montre que les extraits au méthanol de $E$. guineensis, de $C$. prostata, de $E$. indica, de $C$. cajan et de $A$. difformis sont doués d'activité antioxydante. Cependant, l'extrait méthanolique de $E$. guineensis $(95,82 \pm 12,69 \%)$, suivi de celui de $C$. prostata $(94,72 \pm 7,24 \%)$ ont manifesté le plus fort pouvoir antioxydant. Leur $\mathrm{CI}_{50}$ ont été respectivement de 14,20 et de $35,20 \mu \mathrm{g} / \mathrm{mL}$. $E$. indica $(82,19 \pm 10,14 \%)$ a révélé une activité intermédiaire avec une $\mathrm{CI}_{50}$ de $46,00 \mu \mathrm{g} / \mathrm{mL}$ (Tableau 3). Tandis que ceux de $C$. cajan $(53,77 \pm 9,93 \%$ ) et $A$. difformis $(51,78 \pm 12,11 \%)$ ont induit une faible activité. Selon les $\mathrm{CI}_{50}$, les extraits les plus actifs sont ceux de E. guineensis et $C$. cajan. Cependant, ils sont moins actifs que le Trolox $\left(\mathrm{CI}_{50}=7,57 \mu \mathrm{g} / \mathrm{mL}\right)$. 
Tableau 4 : Pourcentages d'inhibition de l'ABTS des espèces végétales

\begin{tabular}{lcll}
\hline Espèces végétales et témoin & $\begin{array}{l}\text { Extraits } \\
\text { méthanoliques }\end{array}$ & Extraits hexaniques & Extraits aqueux \\
\hline Anchomanes difformis & $51,78 \pm 12,11^{\mathrm{c}}$ & $11,42 \pm 3,47^{\mathrm{de}}$ & $86,78 \pm 10,56^{\mathrm{a}}$ \\
Cajanus cajan & $53,77 \pm 9,93^{\mathrm{c}}$ & $50,71 \pm 11,82^{\mathrm{c}}$ & $54,61 \pm 13,36^{\mathrm{c}}$ \\
Cyathula prostata & $94,72 \pm 7,24^{\mathrm{a}}$ & $41,67 \pm 12,12^{\mathrm{c}}$ & $93,78 \pm 6,52^{\mathrm{a}}$ \\
Elaeis guineensis & $95,82 \pm 12,69^{\mathrm{a}}$ & $54,77 \pm 11,66^{\mathrm{c}}$ & $84,21 \pm 10,26^{\mathrm{a}}$ \\
Eleusine indica & $82,19 \pm 10,14^{\mathrm{b}}$ & $93,74 \pm 9,32^{\mathrm{a}}$ & $27,07 \pm 10,85^{\mathrm{d}}$ \\
Mimosa pudica & $8,95 \pm 1,06^{\mathrm{de}}$ & $10,13 \pm 1,04^{\mathrm{de}}$ & $8,36 \pm 0,89^{\mathrm{de}}$ \\
Musa paradisiaca & $8,15 \pm 1,07^{\mathrm{de}}$ & $8,19 \pm 1,40^{\mathrm{de}}$ & $7,54 \pm 1,15^{\mathrm{de}}$ \\
Nephrolepis bisserata & $5,42 \pm 0,95^{\mathrm{de}}$ & $9,570 \pm 0,84^{\mathrm{de}}$ & $9,26 \pm 0,80^{\mathrm{de}}$ \\
Sida acuta & $8,69 \pm 1,06^{\mathrm{de}}$ & $10,01 \pm 0,60^{\mathrm{de}}$ & $8,14 \pm 0,85^{\mathrm{de}}$ \\
Triplochiton scleroxylon & $7,97 \pm 0,55^{\mathrm{de}}$ & $9,148 \pm 1,40^{\mathrm{de}}$ & $6,81 \pm 0,94^{\mathrm{de}}$ \\
\hline
\end{tabular}

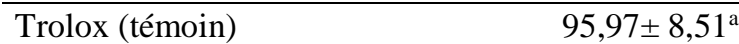

Paramètres statistiques

$\mathrm{F}(4,305)=6,4262: p=0,001$

Les valeurs avec les mêmes lettres en exposant dans la même colonne ne sont pas statistiquement différentes.

${ }^{\mathrm{a}}$ : activité forte, ${ }^{\mathrm{b}}$ : Activité intermédiaire, ${ }^{\text {de }}$ : Activité faible

Pour les extraits aqueux, l'extrait de $C$. prostata $(93,781 \pm 6,52 \%)$ a donné la plus forte activité avec une $\mathrm{CI}_{50}$ de $43,10 \mu \mathrm{g} / \mathrm{mL}$. Par contre, les extraits de A. difformis $(86,64 \pm 10,56)$, et $E$. guineensis $(84,21 \pm 10,26 \%)$ ont exhibé des activités intermédiaires et ont des $\mathrm{CI}_{50}$ respectives de 47,10 et de $50,00 \mu \mathrm{g} / \mathrm{mL}$. Les extraits hexaniques de E. indica, de E. guineensis, de $C$. cajan ont montré des activités antiradicalaires. Toutefois, l'extrait hexanique de E. indica $(93,74 \pm 9,32 \%)$ a donné la plus forte activité. E. guineensis $(54,77 \pm 11,66 \%)$, et $C$. cajan $(54,61 \pm 13,36 \%)$ ont généré des activités intermédiaires. Ces extraits sont moins actifs que le Trolox $(7,57 \mu \mathrm{g} / \mathrm{mL})$, avec des $\mathrm{CI}_{50}$ comprises entre 120,00 et $467,00 \mu \mathrm{g} / \mathrm{mL}$ (tableau 5). En définitive, les extraits les plus actifs sont ceux méthanoliques de $C$. cajan, $C$. prostata, $E$. indica, E. guineensis et A. difformis.

Tableau 5 : Concentrations inhibitrices $\left(\mathrm{CI}_{50}\right)$ en $\left.\mu \mathrm{g} / \mathrm{mL}\right)$ des extraits végétaux testés

\begin{tabular}{lllll}
\hline Espèces végétales et témoins & Organes testés & Extraits & DPPH & ABTS \\
\hline Cyathula prostata & Plante entière & Méthanol & 32,60 & 35,20 \\
Anchomanes difformis & Feuilles & Méthanol & 60,00 & 50,60 \\
Elaeis guineensis & Racines & Méthanol & 41,90 & 14,20 \\
Eleusine indica & Racines & Méthanol & 36,90 & 46,00 \\
Cajanus cajan & Feuilles & Méthanol & 27,20 & 21,50 \\
Cyathula prostata & Feuilles & Eau & 30,00 & 43,10 \\
Anchomanes difformis & Feuilles & Eau & 45,80 & 47,10 \\
Elaeis guineensis & Racines & Eau & $>1000$ & 50,00 \\
Eleusine indica & Racines & Eau & $>1000$ & $>1000$ \\
Cajanus cajan & Feuilles & Eau & $>1000$ & $>1000$ \\
Cajanus cajan & Feuilles & Hexane & $>1000$ & 467,00
\end{tabular}




\begin{tabular}{llllc} 
Anchomanes difformis & Feuilles & Hexane & $>1000$ & $>1000$ \\
Elaeis guineensis & Racines & Hexane & $>1000$ & 147,98 \\
Eleusine indica & Racines & Hexane & $>1000$ & 120,00 \\
Cyathula prostata & Feuilles & Hexane & $>1000$ & $>1000$ \\
Vitamine C & & & 8,34 & nd \\
Trolox & & & nd & 7,57 \\
\hline
\end{tabular}

nd = non déterminé

\section{Discussion}

Ce travail a été mené afin d'évaluer l'activité antiradicalaire de 10 plantes utilisées en cosmétopée par la population de la région de Tiassalé dans les soins et l'entretien de la peau puis d'identifier les composés chimiques susceptibles d'être responsables des effets observés.

Après les extractions, les extraits aqueux ont montré les plus grands rendements, suivis des extraits au méthanol. L'eau est le solvant qui donne les meilleurs rendements. La forte capacité d'extraction de l'eau pourrait justifier le rendement observé et le choix accordé à l'eau comme solvant de préparation des produits cosmétiques en médecine traditionnelle. Par ailleurs, la présence d'eau dans les organes végétaux augmenterait la perméabilité des tissus végétaux et favoriserait le phénomène de diffusion de masse dans l'étape d'extraction (Moure et al., 2000 ; Durant et al., 2011).

Tous les extraits méthanoliques (E. guineensis, E. indica et $C$. cajan, d'E. indica) sont riches en polyphénols, en flavonoïdes, en tanins galliques ainsi qu'en alcaloïdes. Des saponosides ont été aussi détectées chez certains extraits. Parmi ces espèces, Wei et al., (2013) avaient déjà caractérisé ces mêmes composés dans les feuilles de $C$. cajan, récoltées en Indonésie. Quant à $A$ difformis, les travaux de Békro et al. (2012) ont montré la présence de polyphénols, de flavonoïdes dans les rhizomes récoltés en Côte d'Ivoire. Ceux de Adel et al., (2009) ont mis en évidence des polyphénols, des flavonoïdes dans les feuilles de E. indica en Malaisie. La présence de phytocomposés observée dans ce travail justifierait l'activité antioxydante des espèces végétales testées. Les polyphénols sont considérés comme un groupe majeur de composés qui contribuent aux activités antioxydantes des plantes en tant que piégeurs de radicaux libres en raison de leurs groupes hydroxyles (Nickavar et al., 2008).

Sur un total de 30 extraits évalués pour leur pouvoir antioxydant avec le test antiradicalaire au DPPH, sept dont cinq extraits méthanoliques et deux aqueux ont montré un pourcentage d'inhibition superposable à celui de la Vitamine C $(95,79 \pm 10,53 \%)$. Cependant, les extraits méthanoliques de $C$. cajan $(95,71 \pm 9,67 \%)$, de C. prostata $(94,91 \pm 7,04 \%)$, de E. indica $(93,54 \pm 10,21 \%)$ sont les plus actifs. Ces résultats suggèrent que les extraits aqueux et méthanoliques concentrent le plus de constituants chimiques et 
possèdent la capacité de donner de l'hydrogène à un radical libre. Ce piégeage permet de balayer les dommages potentiels causés par les radicaux libres (Wong et al., 2006 ; Chang et al., 2012). Ils sont en fait un mélange complexe d'acides organiques, d'acides aminés et de sucres qui peuvent contribuer également à la séquestration des radicaux libres électriquement chargés (Wong et al., 2006 ; Chang et al., 2012).

Avec l'ABTS, 11 extraits sont actifs. Les extraits méthanoliques de $E$. guineensis $(95,82 \pm 12,69 \%)$, de C. prostata $(94,72 \pm 7,24 \%)$ sont les plus actifs. Tandis que, l'extrait à l'hexane de $E$. indica $(93,74 \pm 9,32 \%)$ a eu le plus fort potentiel, avec une $\mathrm{CI}_{50}$ de $120,00 \mu \mathrm{g} / \mathrm{mL}$. Ce résultat pourrait indiquer que les composés phénoliques, ainsi que les groupes hydroxyles des plantes ne sont pas les principaux chélateurs et réducteurs présents chez les extraits testés. Des acides gras, des triterpènes, des flavonoïdes aglycones hautement méthoxylés pourraient agir indépendamment ou en synergie. La présence d'autres groupements fonctionnels dans les molécules telles que les doubles liaisons conjuguées et également le caractère hautement nucléophile du noyau aromatique joueraient des rôles importants dans les activités antioxydantes (Amic et al., 2003). Tous ces paramètres permettent une délocalisation des électrons offrant une résonance et une structure stable à cette molécule antioxydant.

Parmi ces plantes testées, l'extrait méthanolique de feuilles de C. cajan est le plus actif avec une $\mathrm{CI}_{50}$ de $27,10 \mu \mathrm{g} / \mathrm{mL}$. Nan et al., (2009) ont rapporté une $\mathrm{CI}_{50}$ de l'ordre de $404,91 \mu \mathrm{g} / \mathrm{mL}$ pour l'extrait d'un échantillon de la plante récolté en Chine. Les travaux de Erkan et al., (2008) ont également montré l'activité antioxydante d'un extrait au méthanol des feuilles de cette même plante. On note qu'un pouvoir antioxydant est rapporté pour cette espèce végétale comme dans la présente étude, même si les $\mathrm{CI}_{50}$ sont différentes. Les feuilles sont couramment utilisées partout dans le monde pour le traitement de diverses maladies comme les lésions cutanées (Nan et al., 2009).

L'extrait méthanolique des feuilles de A. difformis a montré une activité antioxydante intermédiaire, avec une $\mathrm{CI}_{50}$ de $60,00 \mu \mathrm{g} / \mathrm{mL}$. Stephanie et al. (2015) avaient déjà rapporté au Cameroun, l'activité antioxydante des extraits éthanoliques des rhizomes de la plante avec une $\mathrm{CI}_{50} \mathrm{de} 6,49 \mathrm{mg} / \mathrm{mL}$.

L'extrait aqueux de cette plante $(88,54 \pm 7,04 \%)$ a également généré une activité intermédiaire avec une $\mathrm{CI}_{50}$ de $45,80 \mu \mathrm{g} / \mathrm{mL}$. Cet extrait est plus actif que celui au méthanol de cette même plante. Ce résultat est similaire à celui de Moukimou et al. (2014) qui avaient déjà rapporté au Benin une activité antiradicalaire intermédiaire $\left(\mathrm{CI}_{50}=26,50 \mathrm{mg} / \mathrm{mL}\right)$. Cette espèce végétale est utilisée en médicine traditionnelle pour ses propriétés antiradicalaires et curatives dans le traitement de plusieurs maladies dont la gale, les sensations de brûlure dans les pieds, les éruptions cutanées. Le pouvoir antioxydant de l'extrait aqueux justifierait certainement l'usage des 
feuilles de A. difformis en cosmétopée dans les soins de la peau et des phanères. Quant aux racines de E. guineensis et de E. indica à notre connaissance, c'est la première fois que leur pouvoir antiradicalaire est rapporté.

\section{Conclusion}

Le tri phytochimique a révelé que ces extraits renferment de grands groupes de composés chimiques entre autres des polyphénols, des flavonoïdes, des tanins, des alcaloïdes, des saponosides. La présence de ces composés renforce le potentiel antioxydant observé chez les espèces étudiées. Pour les activités antiradiculaires, les résultats obtenus ont montré le pouvoir antioxydant des extraits préparés à partir des 10 plantes utilisées en cosmétopée dans la région de Tiassalé. Les extraits des racines de $E$. guineensis, des racines de $E$. indica, les feuilles de $C$. cajan et des fruits de $C$. prostasta ont manifesté les fortes activités. Des formulations cosmétiques à base d'antioxydants naturels tirés de ces plantes actives seraient bénéfiques et profitables à la population pour l'intégrité et le bien-être de la peau.

\section{Remerciements}

Les auteurs sont reconnaissants à l'Initiative DELTAS Africa [Afrique One-ASPIRE / DEL-15-008] qui soutient le Dr Ahoua Constant pour ses études postdoctorales. Afrique One-ASPIRE est financé par un consortium de donateurs dont l'Académie Africaine des Sciences (AAS), l'Alliance pour l'Accélération de l'Excellence en Science en Afrique (AESA), l'Agence du Nouveau Partenariat pour la Planification et la Coordination du Développement en Afrique (NEPAD), le Wellcome Trust [107753/A/15/Z] et le gouvernement britannique.

\section{References:}

1. Adel S. Al-Z, Ahmad B.A., Siddig I.A., Chew Y.P., Syam M. \& Manal M.E. 2009. Eleucine indica possesses antioxidant, antibacterial and aytotoxic properties. Complementary and Alternative Medicine 8 (1): $1-6$.

2. Amic D., Davidovic-Amic D., Beslo D. \& Trinajstic N. 2003. Structure radical scavenging. Croatia Chemica Acta 76 (1) : 55 - 61.

3. Attoh-Touré H., Ekra K. \& Tiembre I. 2009. Etude de la flore fongique dermatophytique de l'Hôpital Félix Houphouët Boigny. Annales de Dermatologie et de Vénéréologie 10 (1) : 64 -71.

4. Békro M., Boua B.B., Diaby A. \& Bekro Y.A. 2013. Screening phytochimique bio guidé et évaluation in vitro des propriétés purgatives de Anchomanes difformis (Blume) Engl, une plante utilisée 
en Côte d'Ivoire dans le traitement folklorique de la constipation. Nature et Technologie (9) : 20 - 26.

5. Bhate K. \& Williams H.C. 2013. Epidemiology of acné vulgaris. Journal de Mycologie Médicale 168 (3) : 85 - 474.

6. Chang C.J., Tzeng T.F., Liou S.S., Chang Y.S. \& Liu I.M. 2012. Acute and 28-Day Subchronic Oral Toxicity of an Ethanol Extract of Zingiber zerumbet (L.) Smith in Rodents. Evidence-Based Complementary and Alternative Medicine (10) : 2 -11.

7. Dagnelie P. (1999). Théorie et méthode statistique, Presse agronomique de Gembloux, $2^{\mathrm{è}}$ Ed. Gembloux (Belgique), 378- 451.

8. Dohou N., Yani K ., Thahrouch S., Hassani I.L. M., Badoc A. \& Mira G.N. 2005. Screeming phytochimique d'une endémique ibéroMarocaine; Thynelaea lythroides. Bulletin de la Société Pharmaceutique de Bordeaux 142 (4) : 61-78.

9. Durant M., Molinier V., Kunz W. \& Aubry J-M. 2011. Classification of organic solvents revisited by using the COSMO-RS approach. Annual Review of Chemical and Biomolecular Engineering 17(18) : 64 - 5155.

10. Erkan N., Ayranci G. \& Ayranci E. 2008. Antioxidant activities of rosemary (Rosmarinus officinalis L.), blackseed (Nigella sativa L.), essential oil, rosmarinic acid and sesamol. Food Chemistry 110 (1) : 76-82.

11. Favier A. 2013. Le stress oxydant Intérêt conceptuel et expérimental dans la compréhension des mécanismes des maladies et potentiel thérapeutique. L'actualité chimique (270) : 108 -115.

12. Guetchueng S.T., Gbaweng J.Y. \& Tchinda A.T. 2015. Phenolic content and DPPH scavenging activity of Carpolobia luteaand, Anchomanes difformis. International Journal of Pharma Sciences and Research, 6 (5) : 0975-9492.

13. Leon G., Jan S., Robert H.P., Amanda B. \& James L. 1960. Current status of griseofulvin: Report on one hundred seventy-five cases. Journal of the American Medical Association 172, (6), p. 532-538.

14. Mallikharjuna P.B., Rajanna L.N., Seetharam Y.N. \& Sharanabasappa G. K. 2007. Phytochimical studies of Stychnos potatorum L. f, a medical plant. E- Journal of Chemistry (4) : 510-518.

15. Merck E. 1980. Révélateurs pour la chromatographie en couche mince et sur papier. In: Darmstadt (Ed), Paris (France), 153 p.

16. Miller N.J., Rice-Evans C., Davies M.J., Gopinathan V. \& Milner A. 1993. A novel method for measuring antioxidant capacity and its application to monitoring the antioxidant status inpremature neonates. Clinical Sciences 84(4) : 407-412. 
17. Moukimou L., Osseni A., Agbangnan P.D.C., Bossou A., Yedomonhan P.Y., Avlessi F. \& Sohounhloue D. 2014. Activités antiradicalaire et étude des composés volatils de trois plantes de la médecine traditionnelle du Bénin : Anchomanes difformis, Parkia biglobosa et Polyalthia longifolia. International Journal of Innovation and Applied Studies 9 (4) : 1609 -1619.

18. Moure A., Franco D., Sineiro J., Dominguez H., Nunez M.J. \& Lema J. M. 2000. Evaluation of extracts from Gevuina avellana hulls as antioxidants. Journal of Agricultural and Food Chemistry 48 (9) : 3897-3890

19. N'Guessan K., Kadja B., Zirihi G.N., Traoré D. \& Aké-Assi L. 2009. Screening phytochimique de quelques plantes médicinales ivoiriennes utilisées en pays Krobou (Agboville, Côte-d'Ivoire). Science et Nature $6(1): 1-15$.

20. Nickavar B., Alinaghi A. \& Kamalinejad M. 2008. Evaluation des propriétés antioxydantes de cinq espèces de Mentha. Irannian Journal of Pharmaceutical Research 7 (3) : 203 - 209.

21. Sanchez-Moreno C. 2002. Methods used to evaluate the free radical scavenging activity in food and biological systems. Food Science and Technology International 8(3) : 121 -137.

22. Wagner H., Seegert K., Sonnenbichler H., Ilyas M. \& Odenthal K.P. 1987. Steroid Alkaloids of Funtumia africana. Planta Medica 53 (5) : 444 - 449.

23. Wie Z.F., Luo M., Zhao C.J., Li C.Y., Gu C.B., Wang W., Zu Y. G., Efferth T. \& Fu Y.J. 2013. UV-induced changes of active components and antioxidant activity in postharvest pigeon pea [Cajanus cajan (L.) Millsp.] leaves. Journal of Agricultural and Food Chemistry 61 (6) : $1165-1171$.

24. Wong S.P., Leong L.P. \& William J.H. 2006. Antioxidant activities of aqueous extracts of selected plants. Food Chemistry 99 (4) : 775 -783. 